\title{
DSMC SIMULATION OF HIGH MACH NUMBER TAYLOR-COUETTE FLOW
}

\author{
S. Pradhan \\ Department of Chemical Engineering, Indian Institute of Science, Bangalore- 560 012, India
}

\begin{abstract}
The main focus of this work is to characterize the Taylor-Couette flow of ideal gas between two coaxial cylinders at Mach number $M a=\left(U_{-} w / \operatorname{sqrt}\left\{k b \quad T_{-} w / m\right\}\right)$ in the range $0.01<\mathrm{Ma}<30$, and Knudsen number $K n=\left(1 /\left(\backslash \operatorname{sqrt}\{2\} \backslash p i d^{\wedge} 2 \quad n \_d\left(r_{-} 2-r_{-} 1\right)\right)\right)$ in the range $0.001<\mathrm{Kn}<10$, using two-dimensional (2D) direct simulation Monte Carlo (DSMC) simulations [1, 2, 3, 4, 5, 6, 7, 8, 9, 10]. Here, $r_{-} 1$ and $r_{-} 2$ are the radius of inner and outer cylinder respectively, $U \_w$ is the circumferential wall velocity of the inner cylinder, $T \_w$ is the wall temperature, $n \_d$ is the number density of the gas molecules, $m$ and $d$ are the molecular mass and diameter, and $k b$ is the Boltzmann constant. The cylindrical surfaces are specified as being diffusely reflecting with the thermal accommodation coefficient equal to one.

In the present analysis of high Mach number compressible Taylor-Couette flow using DSMC method, wall slip in the temperature and the velocities are found to be significant ((Pradhan \& Kumaran, J. Fluid Mech., vol. 686, 2011, pp. 109-159); (Kumaran \& Pradhan, J. Fluid Mech., vol. 753, 2014, pp. 307- 359)). Slip occurs because the temperature/velocity of the molecules incident on the wall could be very different from that of the wall, even though the temperature/velocity of the reflected molecules is equal to that of the wall. Due to the high surface speed of the inner cylinder, significant heating of the gas is taking place. The gas temperature increases until the heat transfer to the surface equals the work done in moving the surface. The highest temperature is obtained near the moving surface of the inner cylinder at a radius of about (1.26r_1).

In a compressible Taylor-Couette flow we examine the result that the splitting of the Taylor vortices takes place proportional as $\left(L /\left(r_{-} 2-r_{-} l\right)\right)$. The resolution suggested by our simulation is that even though the Mach number based on the wall velocity and temperature is large, the local Mach number based on the local dissipation velocity in regions of high shear decreases due to an increase in temperature. Due to this, the ratio of the mean free path and characteristics flow scale (lambda/(r_2 - r_l $))$ appears to taper off in the high Mach number limit.

A modification of the velocity profile in the viscous rotating boundary layer near the wall, which takes into account temperature and density variations, is derived. The variation of the velocity and temperature is predicted under the assumption that the increase in temperature across the viscous rotating boundary layer is larger than the wall temperature. It is found that the scaling laws do depend on the molecular model, through the dependence of viscosity and thermal conductivity on the temperature. The predicted law, are found to be in good agreement with simulations, for two different molecular models, the hard-sphere and the variable hard-sphere.
\end{abstract}

KEY WORDS: Compressible Taylor-Couette flow, Rarefied gas flow, Direct Simulation Monte Carlo (DSMC) simulations.

\section{REFERENCES:}

[1] Pradhan, S. \& Kumaran, V. 2011 The generalized Onsager model for the secondary flow in a high-speed rotating cylinder. J. Fluid Mech. 686, 109 - 159.

[2] Kumaran, V. \& Pradhan, S. 2014 The generalized Onsager model for a binary gas mixture. J. Fluid Mech. 753, 307 359.

[3] Bird, G.A. 1994 Molecular gas dynamics and the direct simulation of gas flows. Clarendon Press. Oxford.

[4] Bird, G. A. 1998 Recent advances and current challenges for DSMC. Comput. Math. Appl. 35,1-14.

[5] Cercignani, C. 2000 Rarefied Gas Dynamics. Cambridge University Press.

[6] Chapman, S. \& Cowling, T. 1970 The Mathematical Theory of Non-Uniform Gases. 3rd edn. Cambridge University Press.

[7] Rayleigh, Lord 1916 On the dynamics of revolving fluids. Sci. Pap. 6, 447-453.

[8] Usami, M. 1995 Direct simulation Monte Carlo on Taylor vortex flow. In Rarefied Gas Dynamics (ed. J. Harvey \& G. Lord), vol. 1, pp. 389-395. Oxford University Press. 
[9] Taylor, G. I. 1923 Stability of a viscous liquid contained between two rotating cylinders. Phil. Trans. R. Soc. A 223, 289-343.

[10] Stefanov, S. \& Cercignani, C. 1993 Monte Carlo simulation of the Taylor-Couette flow of a rarefied gas. J. Fluid Mech. 256, 199-213.

\section{DSMC SIMULATION RESULTS:}
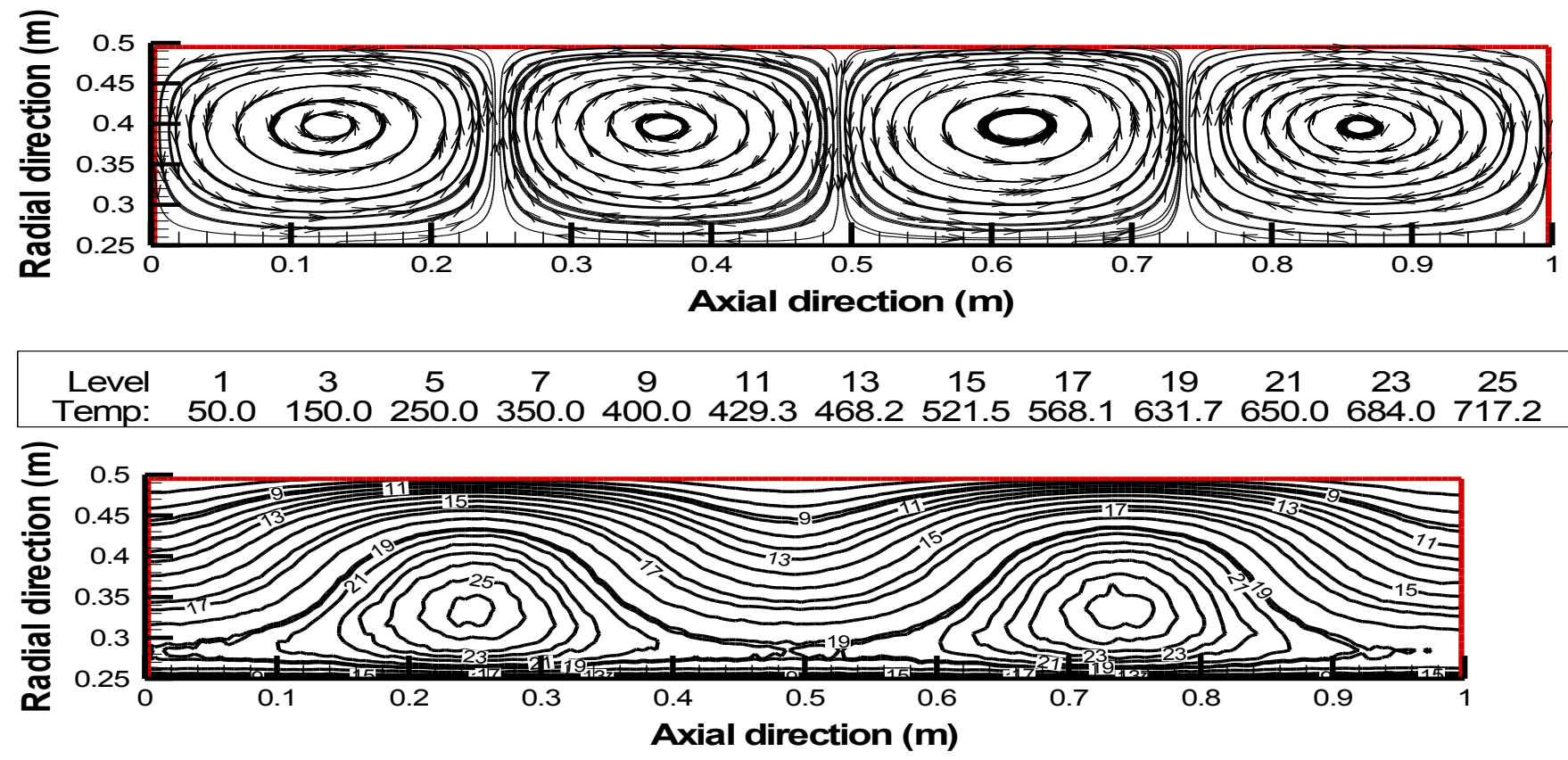

$$
\begin{array}{lccccccc}
\text { Level } & 1 & 4 & 7 & 10 & 13 & 16 & 19 \\
\text { DEN: } & 2.0 \mathrm{E}-06 & 8.0 \mathrm{E}-06 & 1.4 \mathrm{E}-05 & 2.0 \mathrm{E}-05 & 2.6 \mathrm{E}-05 & 3.2 \mathrm{E}-05 & 3.8 \mathrm{E}-05
\end{array}
$$
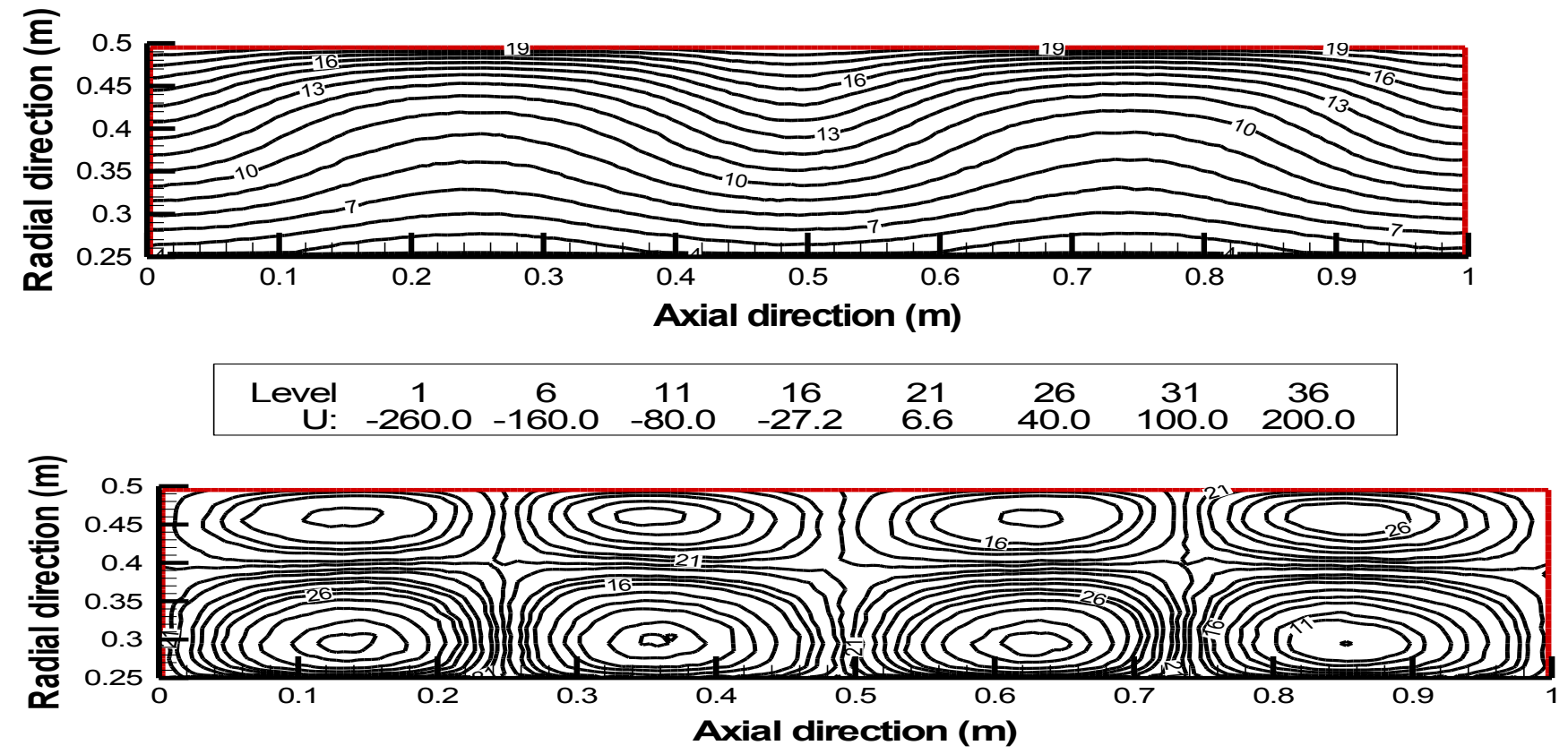

Figure- 1: DSMC simulation results for the Taylor-Couette flow : (a) Streamlines in the flow domain, (b) Temperature contours, (c) Density contours, and (d) Axial-velocity contours in the flow domain. 